\title{
FRAGMENTARY STRUCTURE IN THE L1551 MOLECULAR OUTFLOW
}

\author{
NICHOLAS D. PARKER and GLENN J. WHITE \\ Queen Mary $\mathcal{G}$ Westfield College \\ Mile End Road \\ London $E 1 \& N S$
}

\begin{abstract}
We have obtained a high resolution map of the ${ }^{12} \mathrm{CO} \mathrm{J}=3-2$ emission in the vicinity of HH102 in the L1551 molecular outflow. The data reveal the presence of several bright clumps within the thin shell of low-velocity blue-shifted outflowing gas. There is evidence for further fragmentation in the clumps, as signified by the high derived excitation temperatures and low beam-filling factors. The region of peculiarly energetic activity associated with HH102 coincides with the projected location of impact of a radio jet from IRS5 with the dense ambient gas surrounding the outflow cavity.
\end{abstract}

${ }^{12} \mathrm{CO} \mathrm{J}=3-2$ spectra were obtained using the James Clerk Maxwell Telescope (JCMT). At 345 GHz the beamwidth was $\Theta_{\text {beam }} \sim 15$ arcsec. Figure 1 shows the map of integrated intensity $\left(T_{\mathrm{A}}^{*}\right)$ in the low-velocity blue-shifted gas $\left(v_{\mathrm{lsr}}=5-7 \mathrm{~km}\right.$ $\mathrm{s}^{-1}$, the ambient cloud line-centroid velocity being $v_{\text {lsr }} \sim 7 \mathrm{~km} \mathrm{~s}^{-1}$ ). The thin shell, running roughly northeast - southwest is clearly defined, although this is not the case at larger blue-shifted velocities. The shell separates into three clumps, labelled A, $B$ and $C$, which lie along the locus of peak intensity. A fourth clump, labelled D, is found interior to the arc of the shell. These clumps have size 20 arcsec and a roughly regular spacing of $\sim 55$ arcsec. Thus, since $\Theta_{\text {beam }} \simeq 15$ arcsec, the shell has width of order $20-25$ arcsec over much of its extent. In some places the shell appears narrower than this, perhaps being unresolved, hence $\Theta_{\text {shell }} \lesssim 15$ arcsec. The features seen in these data are consistent with recent high resolution maps obtained using Maximum Entropy image reconstruction (Moriarty-Schieven et al. 1987).

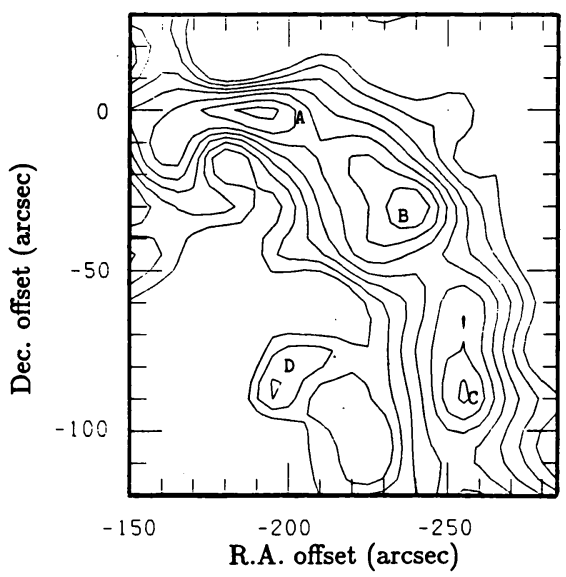

Figure 1. Integrated intensity in the low velocity blue-shifted $\mathrm{CO} \mathrm{J}=3-2$ emission in the vicinity of $\mathrm{HH} 102$.

Uchida et al. (1987) obtained ${ }^{12} \mathrm{CO} \mathrm{J}=1-0$ maps of $\mathrm{L} 1551$ with resolution equal to that of the observations above $\left(\Theta_{\text {beam }} \simeq 16\right.$ arcsec for the NRO $45-\mathrm{m}$ telescope at $\left.115 \mathrm{GHz}\right)$. Comparing the line ratios for the clumps, we find the values of $T_{R}^{*}(3-2) / T_{R}^{*}(1-0)$ are an order of magnitude too low to be consistent with those expected for the hot, optically-thin case. More likely is the optically-thick case, in which (assuming LTE) the excitation temperature , $T_{\text {ex }}$, is obtained from the 
following expression;

$$
\frac{T_{\mathrm{R}}^{*}(3-2)}{T_{\mathrm{R}}^{*}(1-0)} \simeq 3 \frac{e^{h \nu_{10} / k T_{\mathrm{ex}}}-1}{e^{3 h \nu_{10} / k T_{\mathrm{ex}}}-1}
$$

Values of $T_{\text {ex }}$ are found to range from $\sim 25 \mathrm{~K}$ for clump C, up to $\sim 40 \mathrm{~K}$ for clump B. Corresponding beam-filling factors are all considerably less than 1 , with typical values $f \sim 0.2-0.3$. Volume filling factors are smaller again since they scale as $f^{\frac{3}{2}}$. The emergent picture of the blue-shifted outflow at low velocities is one of a thin shell comprising a moderately hot clumpy structure on scales smaller than $\sim 0.01 \mathrm{pc}$ (assuming $D \sim 140 \mathrm{pc}$ to L1551).

Narrow-band $\mathrm{H} \alpha \mathrm{CCD}$ images of the region (Snell et al. 1985) show that the shockexcited emission originates in clumps generally lying interior to the $\mathrm{CO}$ shell (i.e. closer, in projection, to IRS5). If the HH102 region is delineating the cavity - ambient cloud interface then this geometry can be explained as a high-velocity wind being shocked immediately interior to this interface, with the swept up shell lying just behind the shocked region. The HH102 region exhibits velocity dispersions, apparent density enhancements and shock-excited emission signifying a particularly strong interaction of a wind or jet with the surrounding dense molecular material. Figure 2 shows the distribution of high-velocity CS emission (Snell \& Schloerb 1985) together with a VLA map of the $2 \mathrm{~cm}$ radio continuum (Rodriguez 1987).

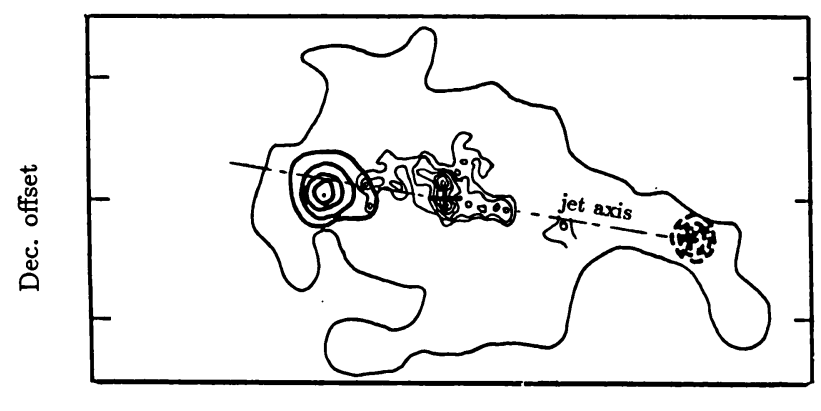

R.A. offset

Figure 2. Contours of $2 \mathrm{~cm}$ radio continuum emission (thin solid contours) overlaid on a map showing the high-velocity CS emission. Thick solid contours are the red-shifted CS integrated intensity and dashed contours are blue-shifted. The maps are on different scales but their relative orientations and $(0,0)$ positions are correct.

Two faint radio 'jets' emanate from IRS5. They are not aligned with the symmetry axis of the outflow, but instead project directly towards the high-velocity CS peaks. We suggest that these jets are responsible for the activity in the $\mathrm{HH} 102$ region, in which the jet material suffers an oblique impact at the boundary of the cavity. Subsequent 'splaying-out' of the flow along the walls of the cavity, coupled with viscous entrainment (Dyson et al. 1988), could then explain the presence of both red- and blue-shifted $\mathrm{CO}$ gas at this location and its equivalent diametrically opposite IRS5. In this case it would not be necessary to invoke the presence of slow rotation of the outflow about its axis (e.g. Uchida et al. 1987) to explain the CO data.

Dyson et al. , 1988. Mass Outflows from Stars and Galactic Nuclei. Kluwer Academic.

Moriarty-Schieven et al. , 1987. Astrophys. J., 319, 742.

Rodriguez, 1987. IAU symp. no. 115, Star Forming Regions. Dordrecht:Reidel.

Snell et al. , 1985. Astrophys. J., 290, 587.

Snell \& Schloerb, 1985. Astrophys. J., 295, 490.

Uchida et al. , 1987. IAU symp. no. 115, Star Forming Regions. Dordrecht:Reidel. 